\title{
Exploring itinerant states in divalent hexaborides using rare-earth $L$ edge resonant inelastic X-ray scattering
}

\author{
Donal Sheets ${ }^{1,2}$, Vincent Flynn ${ }^{1,3}$, Jungho Kim ${ }^{4}$, Mary Upton ${ }^{4}$, Diego Casa ${ }^{4}$, Thomas Gog $^{4}$, Zachary Fisk ${ }^{5}$, Maxim \\ Dzero $^{6}$, Priscilla F. S. Rosa ${ }^{7}$, Daniel G. Mazzone ${ }^{8}$, Ignace Jarrige ${ }^{8}$, Jian-Xin Zhu ${ }^{7}$, and Jason Hancock ${ }^{1,2}$ \\ ${ }^{1}$ Department of Physics, University of Connecticut, Storrs, CT 06269, USA \\ ${ }^{2}$ Institute of Material Science, University of Connecticut, Storrs, CT 06269, USA \\ ${ }^{3}$ Department of Physics and Astronomy, Dartmouth College, Hanover, NH 03755, USA \\ ${ }^{4}$ Advanced Photon Source, Argonne National Laboratory, Argonne, Illinois 60439, USA \\ ${ }^{5}$ Department of Physics, University of California, Irvine, CA 92697, USA \\ ${ }^{6}$ Department of Physics, Kent State University, Kent, OH 44240, USA \\ ${ }^{7}$ Los Alamos National Laboratory, New Mexico 87545, USA and \\ ${ }^{8}$ National Synchrotron Light Source II, Brookhaven National Laboratory, Upton, NY 11973, USA
}

(Dated: May 27, 2019)

\begin{abstract}
We present a study of resonant inelastic X-ray scattering (RIXS) spectra collected at the rareearth $L$ edges of divalent hexaborides $\mathrm{YbB}_{6}$ and $\mathrm{EuB}_{6}$. In both systems, RIXS-active features are observed at two distinct resonances separated by $\sim 10 \mathrm{eV}$ in incident energy, with angle-dependence suggestive of distinct photon scattering processes. RIXS spectra collected at the divalent absorption peak strongly resemble the unoccupied $5 d$ density of states calculated using density functional theory, an occurrence we ascribe to transitions between weakly-dispersing $4 f$ and strongly dispersing $5 d$ states. In addition, anomalous resonant scattering is observed at higher incident energy, where no corresponding absorption feature is present. Our results suggest the far-reaching utility of $L$-edge RIXS in determining the itinerant-state properties of $f$-electron materials.
\end{abstract}

\section{INTRODUCTION}

Resonant inelastic x-ray scattering (RIXS) is an emerging technique capable of probing electronic structure and collective modes of crystals and films of correlated electron systems. Using resonance enhancement of electronic transitions near X-ray edges, this photon-in/photonout scattering technique permits exploration of collective modes where energy, momentum and polarization changes of the scattered photon deliver vital information on the energetics and quantum numbers of complex materials [1]. Recent prominent applications of RIXS mainly focus on transition metal oxides and include the direct observation of charge density waves in high- $T_{c}$ superconductors 2, Mott gap dispersion in their parent compounds 3 5, residual paramagnon fluctuations in doped cuprates [6], and detailed measurement of magnetic and electronic structure in thin films [7] and interfaces [8]. Sustained synchrotron endstation developments have permitted an expanded inquiry into the spin and charge spectra of a broader class of materials which harbor interesting physical phenomena.

Rare earth hexaborides form in the cubic CsCl type lattice structure and exhibit diverse, interesting, and wellcharacterized electronic and magnetic ground states such as putative topological Kondo insulating state in $\mathrm{SmB}_{6}$ [9], superconductivity in $\mathrm{LaB}_{6}$ 10, hidden quadropolar order in $\mathrm{CeB}_{6}$ [1], and colossal magnetoresistance in ferromagnetic $\mathrm{EuB}_{6}[12$. In order to address the systematic variations among this class of materials which underpin their ground states, we focus our attention on two representative divalent systems: nonmagnetic $\mathrm{YbB}_{6}$ and ferromagnetic $\mathrm{EuB}_{6} \cdot \mathrm{YbB}_{6}$ is a non-magnetic small-gap semiconductor 13 , whereas $\mathrm{EuB}_{6}$ is a magnetic semicon- ductor with a ferromagnetic ordering transition at $15.5 \mathrm{~K}$ 14-18. In each case, the covalent bonds among B atoms forms an octahedral cage with a complex valence structure and the $R$ ions sit at sites of cubic symmetry [17, 19. Rare earth $L$-edge RIXS investigations are relatively uncommon but recent success in detecting a quasi-gap and dramatic band reconstruction at the valence transition in $\mathrm{YbInCu}_{4}$ [20] as well as recent activity assessing the topological status of $\mathrm{YbB}_{6}[18,19,21,25]$ has motivated our systematic investigation presented here.

\section{EXPERIMENTAL DETAILS}

RIXS measurements were performed at the MERIX spectrometer at the Advanced Photon Source at Argonne National Lab 26. Here we investigate the $L_{2}$ edge of $\mathrm{YbB}_{6}$ and $L_{3}$ edge of $\mathrm{EuB}_{6}$ while varying incident energy and polarization conditions, culminating in a rather exhaustive report of the RIXS scattering opportunities in divalent rare earth materials. Undulator magnets produce horizontally polarized photons whose bandwidth are then narrowed using two pairs of monochromator crystals. Two Kirkpatrick-Baez mirrors deliver a focused 40 $\times 10 \mu \mathrm{m}$ spot on the sample. Inelastically scattered photons emerging from the scattering volume are collected by a spherical diced crystal analyzer cut from a silicon substrate on a $2 \mathrm{~m}$ arm and backscattered onto a position sensitive micro-strip detector. Overall, energy resolutions of $75 \mathrm{meV}\left(L_{2} \mathrm{YbB}_{6}\right)$ or $140 \mathrm{meV}\left(L_{3} \mathrm{EuB}_{6}\right)$ were achieved. The $\mathrm{YbB}_{6}$ single crystals where grown by the Al-Flux method as described previously [27].

The MERIX spectrometer [26] has three independent axes of rotation on the sample position as well as two 


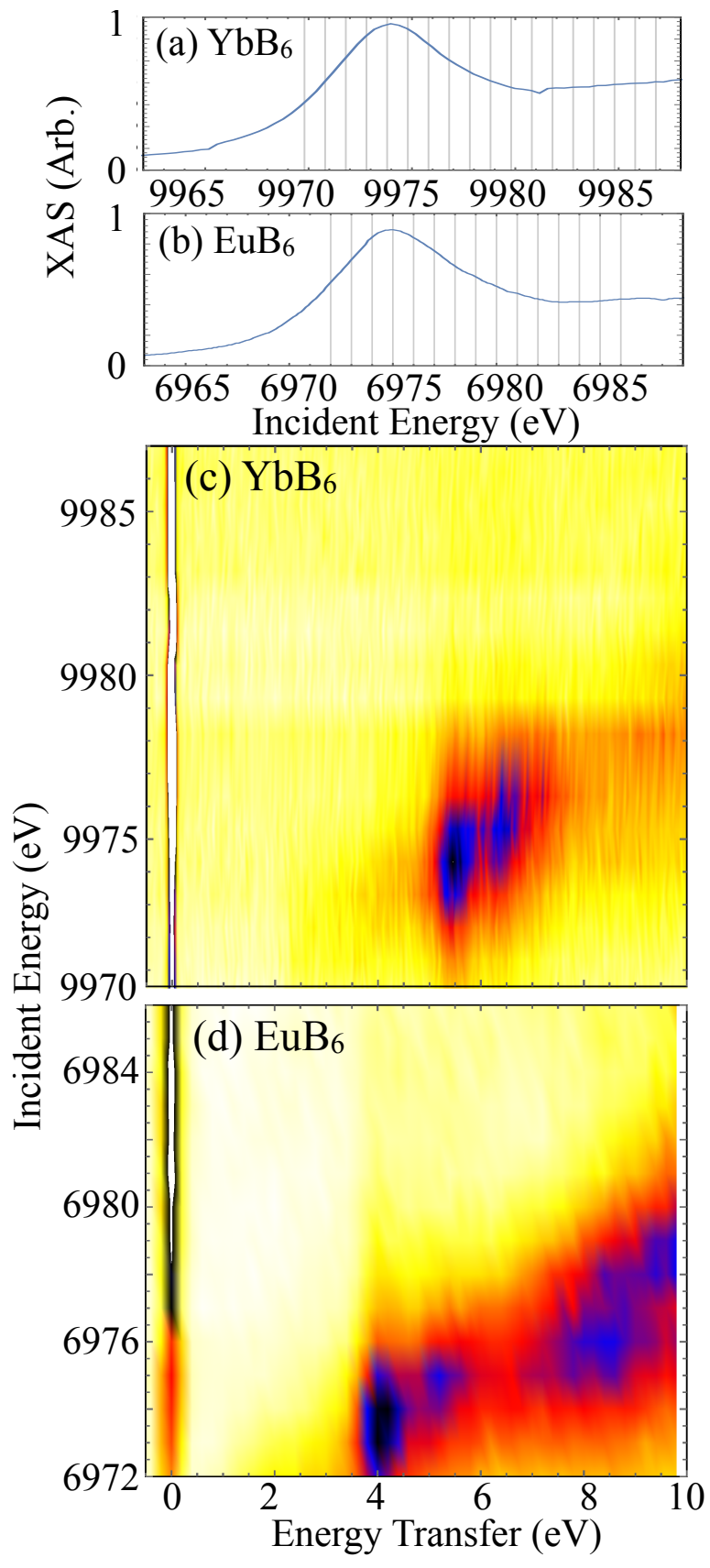

FIG. 1. X-ray absorption spectrum for (a) the $L_{2}$ edge of $\mathrm{YbB}_{6}$ and (b) the $L_{3}$ edge of $\mathrm{EuB}_{6}$. The vertical lines indicate the incident energies selected for the RIXS spectra below. (c) shows the RIXS spectrum for $\mathrm{YbB}_{6}$ as a function of incident photon energy and energy transfer in the depolarized scattering geometry. (d) RIXS spectrum for $\mathrm{EuB}_{6}$ in the depolarized scattering geometry.

axes for the analyzer/detector arm. This flexible geometry allows both horizontal and vertical scattering planes, permitting both angle and energy resolution of the RIXS spectrum and the transverse polarization of the incident photons permits polarization analysis in the scattering process [26]. The momentum transfer is given by $\vec{Q}=$ $\vec{k}_{i}-\vec{k}_{f}$ where $\vec{k}_{i}$ is the incident photons and $\vec{k}_{f}$ is the scattered photon, indexed by the Miller indices $(H K L)$ of the simple cubic lattice with a seven-atom unit cell.

$\mathrm{X}$-ray absorption spectroscopy (XAS) was collected in total fluorescence yield mode with an energy-integrating detector at 90 degrees from the sample to reduce the elastically scattered background while the incident energy from an attenuated beam directly from the monochromator is scanned.

\section{EXPERIMENTAL RESULTS AT THE DIVALENT RESONANCE}

Figure 1 compares the X-ray absorption spectra (XAS) at the $L_{2}\left(2 p_{1 / 2} \rightarrow 5 d\right)$ edge of $\mathrm{YbB}_{6}$ and the $L_{3}$ $\left(2 p_{3 / 2} \rightarrow 5 d\right)$ edge of $\mathrm{EuB}_{6}$. In each case we observe a similar prominent divalent peak and a complete lack of trivalent peak, expected about $7 \mathrm{eV}$ higher in incident energy as observed in mixed rare earth systems [20, 23, 28, confirming the divalency of each compound. Consistent with the magnetic response of each material, the XAS data support the divalency of $\mathrm{YbB}_{6}\left(f^{14}\right)$ and $\mathrm{EuB}_{6}\left(f^{7}\right)$ and imply that each have zero orbital angular momentum associated with the $f$-electron degree of freedom.

The RIXS spectra for the $L_{2}$ edge of $\mathrm{YbB}_{6}$ and $L_{3}$ edge of $\mathrm{EuB}_{6}$ at incident energies represented in Fig. 1 $1 \mathrm{a}, \mathrm{b}$ is shown in Fig. 11,d as a color plot and Fig. 2a,c as line scans. These spectra were collected in the depolarized scattering geometry with momentum transfer $\vec{Q}=(4.75$, $0,0)$ at $T=300 \mathrm{~K}$. In this geometry, the polarization of the scattered photon on the incident one is necessarily zero, a point we return to later. Further, this geometry minimizes the nonresonant elastic (Thomson) scattering, permitting resolution of low-energy inelastic features.

Both materials show broad features which resonate at the divalent XAS peak and in both cases we identify a fluorescence tail above $8 \mathrm{eV}$ energy transfer. The rareearth $L$ RIXS process can be considered as resonant Raman scattering dominated by dipole-allowed transitions $(\Delta l \pm 1)$ in a $2 p \rightarrow 5 d \rightarrow 2 p$ scattering event with a strong $2 p$ core hole potential in the intermediate state. The final state, however, does not contain a core hole and the accessible range of net excitation energies persists down to the instrumental resolution $<100 \mathrm{meV}$. Such low energy scales can be thermally populated at ambient conditions and are therefore relevant for material behavior. For these reasons, rare earth $L$ edge RIXS holds great promise to exploring collective modes in correlated electron systems.

In order to establish detailed connections between the RIXS spectrum and underlying electronic structure, we have performed electronic structure calculations using the full-potential linearized augmented plane wave (FPLAPW) method implemented in the WIEN2k package [29. The generalized gradient approximation (GGA) 30] was used for the exchange-correlation functional. The spin-orbit coupling was included in a second variational 

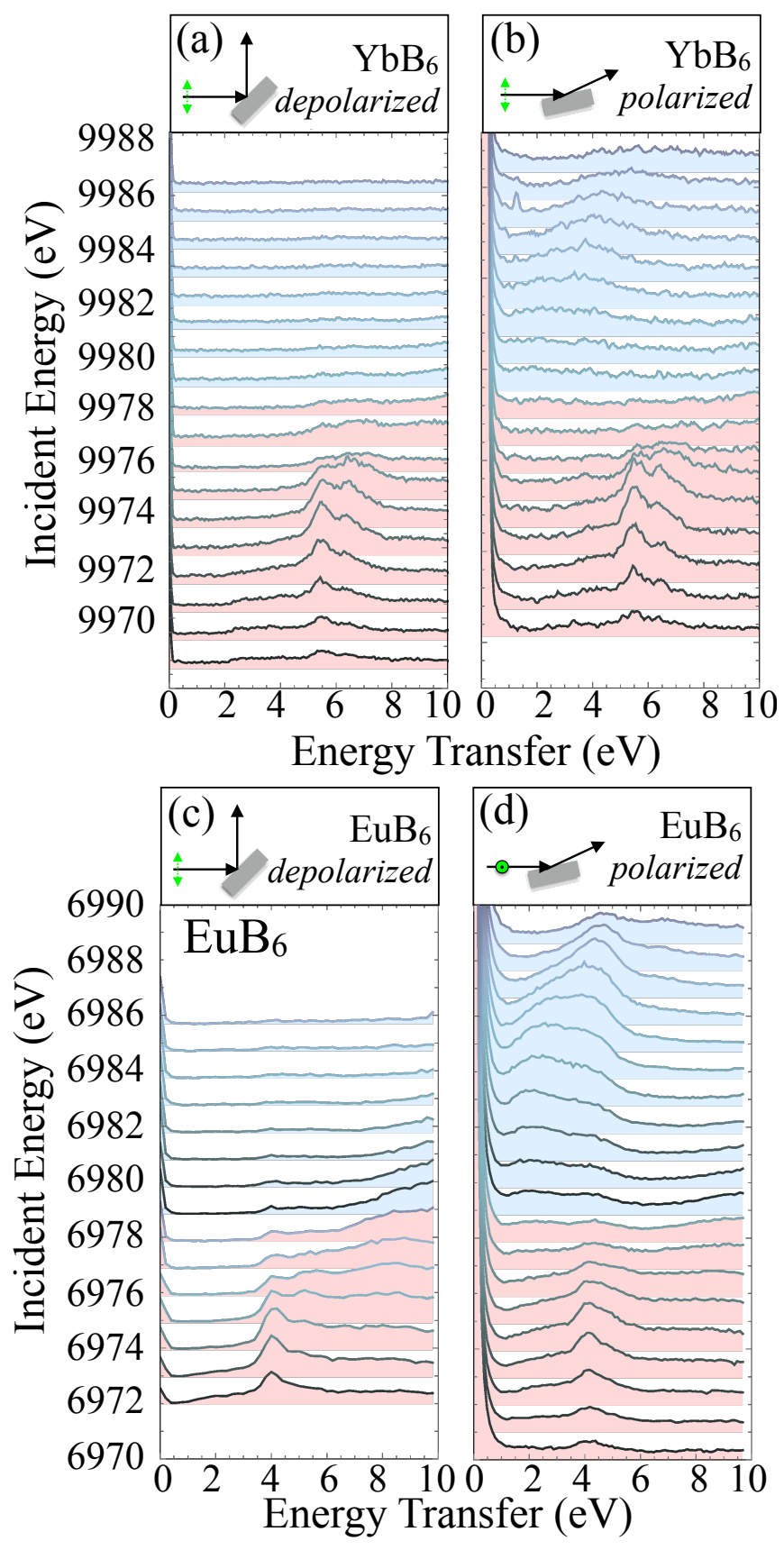

FIG. 2. RIXS data for $\mathrm{YbB}_{6}$ and $\mathrm{EuB}_{6}$ in the depolarized (a, c) and polarized (b, d) scattering geometries at $T=300 \mathrm{~K}$. The insets show the incoming photon polarization as a green arrow while the incident and scattered photon momenta are indicated by black arrows. Pink and blue shading indicate respectively the low and high incident photon energy.

way. The muffin-tin radius $2.5 a_{0}\left(a_{0}\right.$ being the Bohr radius), $2.5 a_{0}$, and $1.57 a_{0}$ and $1.60 a_{0}$ for $\mathrm{Yb}, \mathrm{Eu}$, and $\mathrm{B}$ in $\mathrm{YbB}_{6}$ and $\mathrm{EuB}_{6}$, respectively, and a plane-wave cutoff RKmax $=8$ was taken. Hereafter all theoretical calculations were performed at the experimentally determined lattice constants [14, 31. To address the electronic correlation effects on $\mathrm{Yb}$ - and Eu- $4 f$ electrons, we have
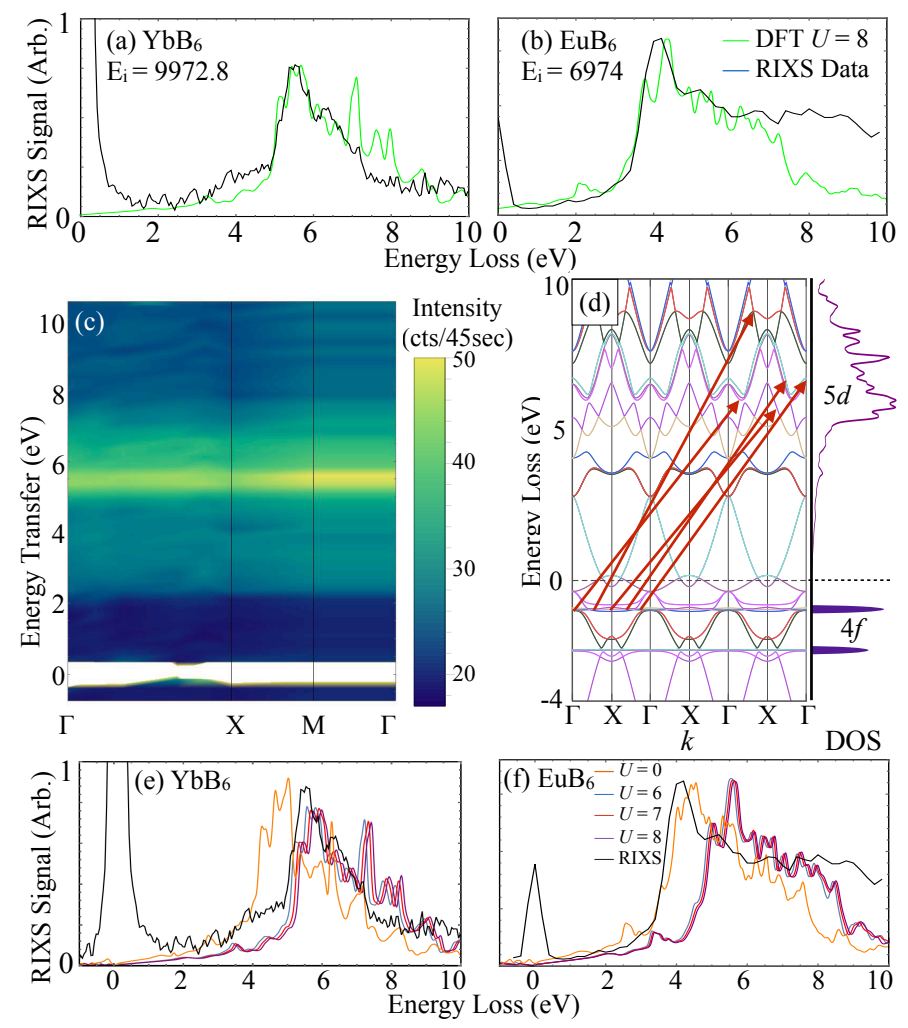

FIG. 3. Comparison of RIXS spectrum with $5 d$ density of states calculations with $U=8$ for (a) $\mathrm{YbB}_{6}$ and (b) $\mathrm{EuB}_{6}$. The RIXS spectra are shown in black with the DFTcalculated $5 d$ density of states in green. In (a) the $\mathrm{YbB}_{6} \mathrm{DOS}$ was downshifted by $.3 \mathrm{eV}$, in (b) the $\mathrm{EuB}_{6}$ DOS was downshifted by $1.2 \mathrm{eV}$. (c) the momentum dependence of the low energy feature in $\mathrm{YbB}_{6}$ along the $\Gamma-\mathrm{X}-\mathrm{M}-\Gamma$ cut, showing weak dispersion. (d) schematic of a set of net $4 f$ to $5 d$ transitions at constant momentum transfer from flat $4 f$ bands to unoccupied $5 d$ states. (e) and (f) comparison of unshifted DFT calculation for $U=0,6,7,8 \mathrm{eV}$ and RIXS spectra. In (e, f) no shift was applied to the DOS.

also performed the $\mathrm{GGA}+U$ calculations by using the self-interaction correction double-counting scheme [32]. We varied the value of the Hubbard repulsion as $U_{\mathrm{e} f}$ $=6,7,8 \mathrm{eV}$. The above choice of Hubbard $U$ values are on par with those reported in earlier work 33, 34. Spin-polarized GGA calculations gives vanishing magnetic moment for $\mathrm{YbB}_{6}$ and $\sim 7 \mu_{B}$ for $\mathrm{EuB}_{6}$, in reasonably good agreement with the experimental measurements [17, 35, 36] and earlier calculations [34.

Although the nonmagnetic state of $\mathrm{YbB}_{6}$ or the moment size of $\mathrm{EuB}_{6}$ are robust against the value of the Hubbard repulsion, the position of $4 f$-orbital energy levels are pushed away below the Fermi energy by this repulsion. In particular, the obtained Yb- $4 f$ level positions for $U_{\mathrm{e} f f}=8 \mathrm{eV}$ are in good agreement with the ARPES measurements on $\mathrm{YbB}_{6}$ [37. Therefore, we present the electronic structure results for this specific value.

Figures $3 a$,b compares the RIXS spectra with the corresponding calculation of the $5 d$ density of states (DOS), 
sampled on a mesh of $15 \times 15 \times 15 \mathrm{k}$ points. The DOS have been shifted in energy to enable detailed comparison of the RIXS line shape and the calculated density of states, including details like the gradual rise below the strong cusp due to a prominent van Hove singularity. The calculation appears to produce sharper features at higher energies, which are likely blurred in the data by a combination of instrumental resolution and coupling to lattice degrees of freedom, as is well-known in optical and RIXS spectra [38, 39. The observed agreement strongly supports an interpretation where electrons are excited into $5 d$ final states from a band of weakly dispersing initial states. We propose here a scattering process wherein electrons originating in the weakly dispersing $4 f$ states are scattering by the core hole potential to $5 d$ final states, illustrated schematically in Fig. $3 \mathrm{~d}$. Because the $f$ band is both occupied and weakly dispersive, the manifold of all such transitions at the momentum set by the scattering geometry provides an image of the unoccupied $5 d$ density of states.

Figure 3. presents RIXS spectra collected along highsymmetry cuts in the simple cubic Brillouin zone of $\mathrm{YbB}_{6}$ at the divalent resonance. Weak dispersion is apparent and supports our interpretation of the scattering process considering that the $4 f$ manifold is completely full and the $5 d$-derived states are nearly empty [37. A similar momentum-independence was observed in $\mathrm{EuB}_{6}$, where similar conditions are present, although in this case, the $4 f$ states are only half full. This situation presents strong contrast to RIXS studies on $5 d$ elements such as Ir and Os, which often display local moment magnetism arising from correlated states within the $5 d$ manifold [40].

Given our interpretation of the rare-earth $L$ egde RIXS spectra collected at the divalent resonance, we consider here the implications for the electronic structure of each material. The van Hove singularity in $\mathrm{YbB}_{6}$ giving rise to the strong cusp is separated energetically from the $4 f$ level by approximately $1.5 \mathrm{eV}$ more than in $\mathrm{EuB}_{6}$. We also note that the bandwidths obtained here from the RIXS data and from the DFT calculation are in good agreement without the need to scale, a step that is often invoked to account for correlation effects [41, 42, which appear to be absent or muted in the unoccupied $5 d$ states of the divalent hexaborides $\mathrm{EuB}_{6}$ and $\mathrm{YbB}_{6}$. Such detailed information on electronic structure with orbital selectivity can serve as a benchmark in the development of new computational schemes in correlated electron systems.

\section{GEOMETRICAL DEPENDENCE OF RIXS PROCESSES}

So far we have considered the RIXS spectra of $\mathrm{YbB}_{6}$ and $\mathrm{EuB}_{6}$ near the divalent resonance taken in the depolarized scattering geometry with scattering angle $2 \theta \simeq$ $90^{\circ}$. In this configuration, the projection of the scattered photon polarization on the incident one is neces-
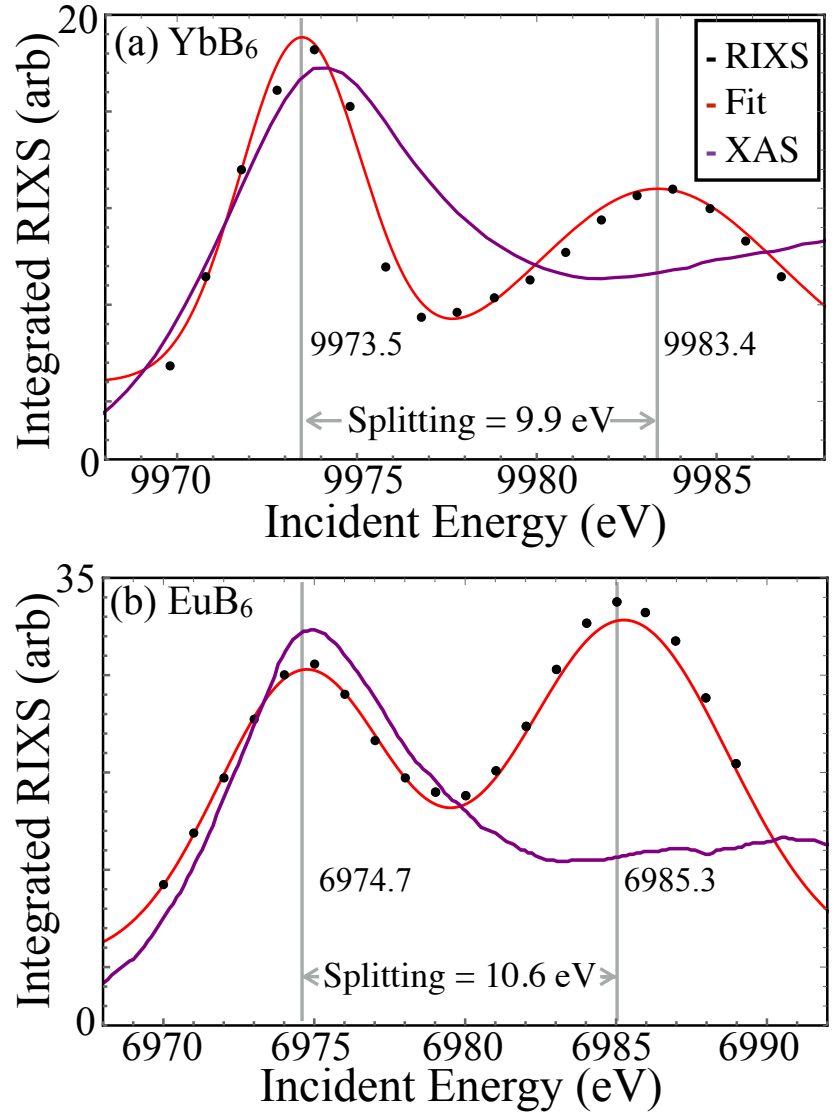

FIG. 4. Total integrated RIXS as a function of incident energy for (a) $\mathrm{YbB}_{6}$ and (b) $\mathrm{EuB}_{6}$. Two peaks are apparent and split by $9.9 \mathrm{eV}$ for $\mathrm{YbB}_{6}$ and $10.6 \mathrm{eV}$ for $\mathrm{EuB}_{6}$. The lower energy peak resonates near the peak in XAS, while the higher energy feature does not have a corresponding peak in the XAS.

sarily zero, constraining the allowed matrix elements and therefore types of excitations observed. We now relax this stringent condition by surveying a polarized scattering condition, illustrated in Fig. 20, d, collected at $\vec{Q}=$ $(1.5,0,0)$ and $2 \theta$ near $25^{\circ}$ at $T=300 \mathrm{~K}$.

As with the depolarized case, we observe a feature resembling the $5 d$ density of states which is strongest at the divalent resonance. In contrast, we also observe a strong set of RIXS transitions at a somewhat higher energy above the divalent peak. For each compound, the high energy feature peaks at a low energy transfer of $\sim 4$ eV (Fig. 4t,d), with a continuum of intensity persisting down to the lowest energies measured. For the case of $\mathrm{EuB}_{6}$, this scattering has been shown to be sensitive to the bulk magnetization of the system. Specifically, below the Curie temperature $T_{c}=15.5 \mathrm{~K}$ [17] a pronounced spectral weight redistribution toward low energy is observed in the RIXS spectra collected at high incident energy. This observation demonstrates the importance of the high-energy resonance because the final states of this scattering process result in excited states which can also be thermally excited and therefore directly influence 

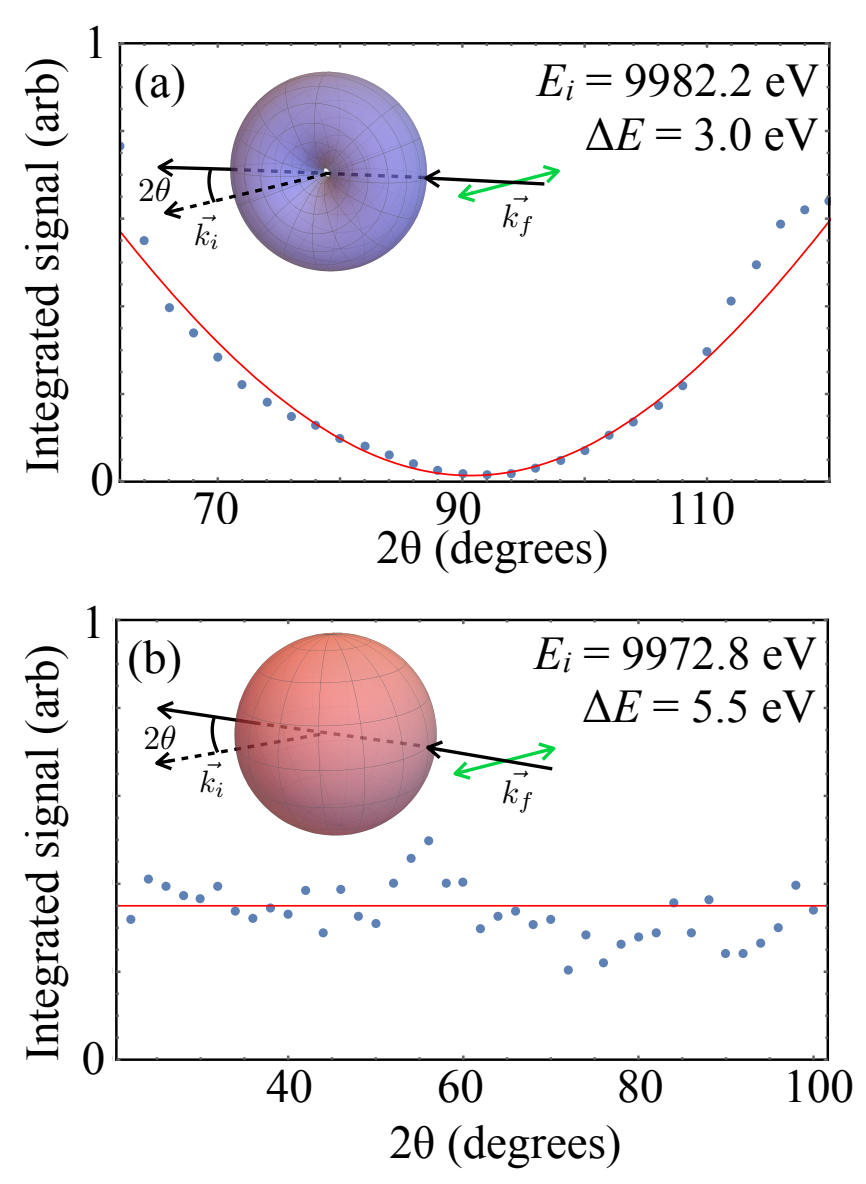

FIG. 5. Theta-two theta scan showing the angle dependence of the two RIXS features in the spectrum of $\mathrm{YbB}_{6}$. (a) shows the angular dependence of the high energy feature at $E_{i}=$ $9982.2 \mathrm{eV}$ with $1.5<\Delta E<4.5 \mathrm{eV}$, showing a clear node at $90^{\circ}$. Red solid line shows a fit to $\cos ^{2}(2 \theta)$. Inset summarizes the observed polarization dependence, scattering geometry and incident polarization vector. (b) shows that the low energy feature at $E_{i}=9972.8 \mathrm{eV}$ with $4 \mathrm{eV}<\Delta E<7 \mathrm{eV}$ has no significant angular dependence, consistent with the horizontal line shown in solid red. The isotropic scattering in this case is represented in the inset as a sphere.

material behavior.

Figures 4 a,b show the total integrated RIXS intensity as a function of incident energy. The lower peak coincides well with the divalent peak in the XAS, while the higher energy peak occurs in a region where the XAS has no pronounced feature. The two-peak structure was fit to two Gaussian profiles plus a constant background to determine the splitting is $\simeq 10 \mathrm{eV}$, which is significantly greater than the documented divalent-trivalent splitting observed to be around $7 \mathrm{eV}[20$. This hidden resonance therefore appears not to coincide with any observable peak in X-ray absorption and furthermore cannot be attributed to a remanent trivalent state.

The scattering intensity produced at high incident energies is absent in the depolarized geometry, an observation we explore and quantify here for $\mathrm{YbB}_{6}$. Figure $5 \mathrm{a}$ displays a theta-two theta angular scan with $E_{i}=9982.2$ $\mathrm{eV}$ and the energy transfer integrated over a region between 1.5 and $4.5 \mathrm{eV}$ to represent the RIXS spectral weight of the high energy resonance. Such a scan corresponds to a linear pathway in reciprocal space which traverses many Brillouin zones while the polarization undergoes smooth variations throughout the scan, showing clearly the behavior arises from polarization effects. Also shown is a fit to a simple angular-dependent form $\cos ^{2} 2 \theta$, reminiscent of Thomson scattering. Such an angular dependence has been observed before at the $K$ edge of $\mathrm{Cu}$ in zero-dimensional cuprate $\mathrm{CuB}_{2} \mathrm{O}_{4}$ [38, which corresponds to an indirect scattering process, discussed below.

The angle dependence of the $4 f \rightarrow 5 d$ excitation produced at the divalent resonance is shown in Fig. 5b, and can be well described as being totally independent of scattering angle. This scattering distribution over angle can be represented as a sphere, shown schematically in the inset of Fig. 5b. This angle independent scattering is consistent with an attribution of a indirect $4 f$ transition to a $5 d$ band with small occupation.

\section{DISCUSSION}

In an indirect RIXS scattering process, the photoexcited electron enters electronic orbitals far above the Fermi level and plays only a small part in generating the excitations, which are dominated by the strong core hole potential. For indirect $\mathrm{Cu} K$ RIXS, theoretical models which neglect the interaction of the $4 p$ "spectator" electron provide adequate description of RIXS spectra in cuprates [1, 4, 38, 43, which are driven by the $1 s$ core hole potential. For the rare-earth $L$ edge RIXS considered here, the $5 d$ states play the role of the spectator and $2 p$ core holes generate the excitations.

Though we have made compelling progress in interpreting the nature of the excitations generated at the divalent resonance, spectra collected at higher energy present significant challenges. We note that the numerical value of the splitting between the peaks of RIXS is rather large: in $\mathrm{YbB}_{6}$, two peaks in the integrated RIXS spectrum are separated by $9.9 \mathrm{eV}$, while for $\mathrm{EuB}_{6}$ there is a splitting of $10.6 \mathrm{eV}$ between the peaks. Rare-earth hexaborides have long been studied and are in use as thermionic emitters due to their low work function and high melting point. For each case presented here, the resonance splitting far exceeds the work function of each material $\sim 4 \mathrm{eV}$ [4], suggestive that to the extent that the fermi level is measured by the divalent peak, the intermediate states generating the high energy peak are unbound from the material. The intermediate states do not then correspond to final states of XAS, but instead correspond to the final states of photoemission. Further work is needed to fully explore the implications of the scenario. 


\section{CONCLUSIONS}

Our resonant inelastic X-ray scattering investigation of the $L$ edges of rare-earth hexaborides $\mathrm{YbB}_{6}$ and $\mathrm{EuB}_{6}$ shows two strong resonant features attributable to distinct RIXS scattering processes. We present compelling evidence that an indirect RIXS feature present at the divalent peak of x-ray absorption has negligible polarization and momentum dependence is due to a net $4 f \rightarrow 5 d$ transition. Quantitative considerations of this feature provide microscopic energy scales such as the $5 d$ bandwidth, energy of the $4 f$ electronic bands, and systematic variation across materials. In addition, we observe a second set of features resonant at a higher incident energy where no peak in absorption can be identified. This feature has strong polarization dependence resembling that of Thompson scattering. Our results suggest far-reaching utility of $L$ edge RIXS experiments in deciphering mixed valent, heavy fermion, hidden order, and other complex phenomenology of $f$-electron materials at a microscopic level. In particular, extension of our quantitative rareearth $L$ edge RIXS work and relation to the underlying electronic structure to more complex systems such as $\mathrm{SmB}_{6}$ and $\mathrm{YbB}_{12}$ hold strong promise toward evalu- ation of the topological status of these and related $3 \mathrm{D}$ materials.

\section{ACKNOWLEDGEMENTS}

Work at the University of Connecticut and Kent State University was supported by the U.S. Department of Energy, Office of Science, Office of Basic Energy Sciences, under Award No. de-sc0016481. This research used resources of the Advanced Photon Source, a U.S. Department of Energy (DOE) Office of Science User Facility operated for the DOE Office of Science by Argonne National Laboratory under Contract No. DE-AC0206CH11357. Daniel G. Mazzone acknowledges funding from the Swiss National Science Foundation, Fellowship No. P2EZP2-175092. Work at Los Alamos National Laboratory was supported by U.S. DOE BES Core Program E3B5. Sample synthesis at Los Alamos was supported by the U.S. DOE BES program "Quantum Fluctuations in Narrow Band Systems". Work at Kent State University by Maxim Dzero was financially supported in part by NSF-DMR-1506547.
[1] L. J. P. Ament, M. van Veenendaal, T. P. Devereaux, J. P. Hill, and J. van den Brink, Rev. Mod. Phys. 83, 705 (2011)

[2] G. Ghiringhelli, M. Le Tacon, M. Minola, S. BlancoCanosa, C. Mazzoli, N. B. Brookes, G. M. De Luca, A. Frano, D. G. Hawthorn, F. He, T. Loew, M. Moretti Sala, D. C. Peets, M. Salluzzo, E. Schierle, R. Sutarto, G. A. Sawatzky, E. Weschke, B. Keimer, and L. Braicovich, Science (New York, N.Y.) 337, 821 (2012)

[3] P. Abbamonte, C. Burns, E. Isaacs, P. Platzman, L. Miller, S. Cheong, and M. Klein, Physical Review Letters 83, 860 (1999)

[4] M. Z. Hasan, E. D. Isaacs, Z.-X. Shen, L. L. Miller, K. Tsutsui, T. Tohyama, and S. Maekawa, Science 288, $1811(2000)$

[5] G. Chabot-Couture, J. N. Hancock, P. K. Mang, D. M. Casa, T. Gog, and M. Greven, Phys. Rev. B 82, 035113 (2010)

[6] M. Le Tacon, G. Ghiringhelli, J. Chaloupka, M. M. Sala, V. Hinkov, M. W. Haverkort, M. Minola, M. Bakr, K. J. Zhou, S. Blanco-Canosa, C. Monney, Y. T. Song, G. L. Sun, C. T. Lin, G. M. De Luca, M. Salluzzo, G. Khaliullin, T. Schmitt, L. Braicovich, and B. Keimer, Nature Physics 7, 725 (2011).

[7] M. P. M. Dean, R. S. Springell, C. Monney, K. J. Zhou, J. Pereiro, I. Božović, B. Dalla Piazza, H. M. Rønnow, E. Morenzoni, J. van den Brink, T. Schmitt, and J. P. Hill, Nature materials 11, 850 (2012).

[8] K.-J. Zhou, M. Radovic, J. Schlappa, V. Strocov, R. Frison, J. Mesot, L. Patthey, and T. Schmitt, Physical Review B 83, 201402 (2011).

[9] M. Dzero, J. Xia, V. Galitski, and P. Coleman, Annual Review of Condensed Matter Physics 7, 249 (2016).
[10] G. Schell, H. Winter, H. Rietschel, and F. Gompf, Phys. Rev. B 25, 1589 (1982)

11] A. Koitzsch, N. Heming, M. Knupfer, B. Büchner, P. Y. Portnichenko, A. V. Dukhnenko, N. Y. Shitsevalova, V. B. Filipov, L. L. Lev, V. N. Strocov, J. Ollivier, and D. S. Inosov, Nature Communications 7, 10876 EP (2016)

[12] R. S. Manna, P. Das, M. de Souza, F. Schnelle, M. Lang, J. Müller, S. von Molnár, and Z. Fisk, Phys. Rev. Lett. 113, 067202 (2014)

[13] J. M. Tarascon, J. Etourneau, P. Dordor, P. Hagenmuller, M. Kasaya, and J. M. D. Coey, Journal of Applied Physics 51, 574 (1980).

[14] M. K. Blomberg, M. J. Merisalo, M. M. Korsukova, and V. N. Gurin, Journal of Alloys and Compounds 217, 123 (1995)

[15] G. A. Wigger, R. Monnier, H. R. Ott, D. P. Young, and Z. Fisk, Phys. Rev. B 69, 125118 (2004).

[16] J. Kim, W. Ku, C.-C. Lee, D. S. Ellis, B. K. Cho, A. H. Said, Y. Shvyd 'ko, and Y.-J. Kim, Physical Review B 87 (2013), 10.1103/PhysRevB.87.155104

[17] S. Süllow, I. Prasad, M. Aronson, J. Sarrao, Z. Fisk, D. Hristova, A. Lacerda, M. Hundley, A. Vigliante, and D. Gibbs, Physical Review B 57, 5860 (1998).

[18] C.-J. Kang, J. D. Denlinger, J. W. Allen, C.-H. Min, F. Reinert, B. Y. Kang, B. K. Cho, J.-S. Kang, J. H. Shim, and B. I. Min, Phys. Rev. Lett. 116, 116401 (2016).

[19] S. Kimura, T. Nanba, M. Tomikawa, S. Kunii, and T. Kasuya, Phys. Rev. B 46, 12196 (1992).

[20] I. Jarrige, A. Kotani, H. Yamaoka, N. Tsujii, K. Ishii, M. Upton, D. Casa, J. Kim, T. Gog, and J. Hancock, Physical Review Letters 114, 126401 (2015). 
[21] M. Xia, J. Jiang, Z. R. Ye, Y. H. Wang, Y. Zhang, S. D. Chen, X. H. Niu, D. F. Xu, F. Chen, X. H. Chen, B. P. Xie, T. Zhang, and D. L. Feng, Scientific reports 4, 5999 (2014)

[22] N. Xu, C. E. Matt, E. Pomjakushina, J. H. Dil, G. Landolt, J. Z. Ma, X. Shi, R. S. Dhaka, N. C. Plumb, M. Radovic, V. N. Strocov, T. K. Kim, M. Hoesch, K. Conder, J. Mesot, H. Ding, and M. Shi, Arxiv Preprint , 1405.0165 (2014), arXiv:1405.0165

[23] Y. Zhou, D.-J. Kim, P. Ferrari, S. Rosa, Q. Wu, J. Guo, S. Zhang, Z. Wang, D. Kang, W. Yi, Y. Li, X. Li, J. Liu, P. Duan, M. Zi, X. Wei, Z. Jiang, Y. Huang, Y.-F. Yang, Z. Fisk, L. Sun, and Z. Zhao, Rapid Communications Physical Review B 92, 241118 (2015)

[24] M. Neupane, S.-Y. Xu, N. Alidoust, G. Bian, D. Kim, C. Liu, I. Belopolski, T.-R. Chang, H.-T. Jeng, T. Durakiewicz, H. Lin, A. Bansil, Z. Fisk, and M. Hasan, Physical Review Letters 114, 016403 (2015).

[25] S. V. Ramankutty, N. De Jong, Y. K. Huang, B. Zwartsenberg, F. Massee, T. V. Bay, M. S. Golden, and E. Frantzeskakis, Journal of Electron Spectroscopy and Related Phenomena 208, 43 (2016)

[26] Y. V. Shvyd 'ko, J. P. Hill, C. A. Burns, D. S. Coburn, B. Brajuskovic, D. Casa, K. Goetze, T. Gog, R. Khachatryan, J.-H. Kim, C. N. Kodituwakku, M. Ramanathan, T. Roberts, A. Said, H. Sinn, D. Shu, S. Stoupin, M. Upton, M. Wieczorek, and H. Yavas, Journal of Electron Spectroscopy and Related Phenomena 188, 140 (2013)

[27] Z. Fisk and J. Remeika, Handbook on the Physics and Chemistry of Rare Earths 12, 53 (1989).

[28] I. Jarrige, H. Yamaoka, J.-P. Rueff, J.-F. Lin, M. Taguchi, N. Hiraoka, H. Ishii, K. D. Tsuei, K. Imura, T. Matsumura, A. Ochiai, H. S. Suzuki, and A. Kotani, Phys. Rev. B 87, 115107 (2013)

[29] P. K. Blaha Karlheinz Schwarz Georg H Madsen Dieter Kvasnicka Joachim Luitz Robert Laskowski Fabien Tran Laurence D Marks, P. Blaha, K. Schwarz, G. K. H Madsen, D. Kvasnicka, J. Luitz, R. Laskowski, F. Tran, and L. D. Marks, WIEN2k An Augmented Plane Wave Plus Local Orbitals Program for Calculating Crystal Properties, Tech. Rep. (2001).

[30] J. P. Perdew, K. Burke, and M. Ernzerhof, Phys. Rev. Lett. 77, 3865 (1996)

[31] A. Bolgar, V. Muratov, A. Blinder, A. Kryklya, and A. Suodis, Journal of Alloys and Compounds 201, 127
(1993)

[32] V. I. Anisimov, I. V. Solovyev, M. A. Korotin, M. T. Czyżyk, and G. A. Sawatzky, Phys. Rev. B 48, 16929 (1993)

[33] J. Kuneš and W. E. Pickett, Phys. Rev. B 69, 165111 (2004)

[34] D. B. Ghosh, M. De, and S. K. De, (2004), arXiv:0406706v1 [arXiv:cond-mat].

[35] J. Tarascon, J. Soubeyroux, J. Etourneau, R. Georges, J. Coey, and O. Massenet, Solid State Communications 37, 133 (1981)

[36] Z. Fisk, D. C. Johnston, B. Cornut, S. von Molnar, S. Oseroff, and R. Calvo, Journal of Applied Physics 50, 1911 (1979)

[37] M. Neupane, S.-Y. Xu, N. Alidoust, G. Bian, D. J. Kim, C. Liu, I. Belopolski, T.-R. Chang, H.-T. Jeng, T. Durakiewicz, H. Lin, A. Bansil, Z. Fisk, and M. Z. Hasan, 10.1103/PhysRevLett.114.016403.

[38] J. Hancock, G. Chabot-Couture, Y. Li, G. Petrakovski, K. Ishii, I. Jarrige, J. Mizuki, T. Devereaux, and M. Greven, Physical Review B 80, 092509 (2009)

[39] J. N. Hancock, G. Chabot-Couture, and M. Greven, New Journal of Physics 12, 033001 (2010)

[40] S. Calder, J. G. Vale, N. A. Bogdanov, X. Liu, C. Donnerer, M. H. Upton, D. Casa, A. H. Said, M. D. Lumsden, Z. Zhao, J.-Q. Yan, D. Mandrus, S. Nishimoto, J. Van Den Brink, J. P. Hill, D. F. Mcmorrow, and A. D. Christianson, Nature Communications 7 (2016), 10.1038/ncomms11651.

[41] Z. K. Liu, M. Yi, Y. Zhang, J. Hu, R. Yu, J.-X. Zhu, R.H. He, Y. L. Chen, M. Hashimoto, R. G. Moore, S.-K. Mo, Z. Hussain, Q. Si, Z. Q. Mao, D. H. Lu, and Z.-X. Shen, Phys. Rev. B 92, 235138 (2015).

[42] M. Kobayashi, K. Yoshimatsu, E. Sakai, M. Kitamura, K. Horiba, A. Fujimori, and H. Kumigashira, Phys. Rev. Lett. 115, 076801 (2015)

[43] C.-C. Chen, B. Moritz, F. Vernay, J. N. Hancock, S. Johnston, C. J. Jia, G. Chabot-Couture, M. Greven, I. Elfimov, G. A. Sawatzky, and T. P. Devereaux, Phys. Rev. Lett. 105, 177401 (2010)

[44] L. Wang, G. Luo, D. Valencia, C. H. Sierra Llavina, R. F. Sabirianov, J. Lu, J.-Q. Lu, W.-N. Mei, and C. Li Cheung, Journal of Applied Physics 114, 143709 (2013). 